\title{
Robotic or laparoscopic surgery for rectal cancer - which is the best answer? A comprehensive review of oncological outcomes
}

\author{
Amandeep Ghuman, Sandra Kavalukas, Steven D. Wexner \\ Department of Colorectal Surgery, Cleveland Clinic Florida, Weston, FL 33331, USA.
}

Correspondence to: Dr. Steven D. Wexner, Department of Colorectal Surgery, Cleveland Clinic Florida, Weston, FL 33331, USA. E-mail:wexners@ccf.org

\begin{abstract}
How to cite this article: Ghuman A, Kavalukas S, Wexner SD. Robotic or laparoscopic surgery for rectal cancer - which is the best answer? A comprehensive review of oncological outcomes. Mini-invasive Surg 2020;4:84.
\end{abstract}

http://dx.doi.org/10.20517/2574-1225.2020.88

Received: 15 Sep 2020 First Decision: 30 Oct 2020 Revised: 2 Nov 2020 Accepted: 6 Nov 2020 Published: 20 Nov 2020

Academic Editor: Sergio W. Larach Copy Editor: Cai-Hong Wang Production Editor: Jing Yu

\begin{abstract}
Treatment of rectal cancer is ever evolving with the introduction of newer surgical technologies and multimodal treatment approach. The literature evaluating the various surgical treatment options with regards to operative and nonoperative outcomes is abundant. This is a comprehensive review focused on oncological outcomes of rectal cancer resection performed robotically or laparoscopically. Based on the current literature available, there is no significant difference in total mesorectal excision completeness, lymph node harvest, positive circumferential resection margin, or proximal resection margin between robotic and laparoscopic approaches for rectal resection. Selection of surgical approach should not be based on pathological outcomes as they are equivalent.
\end{abstract}

Keywords: Robotic, robotics, laparoscopic, laparoscopy, rectal cancer, rectal carcinoma, total mesorectal excision

\section{INTRODUCTION}

The treatment of rectal cancer has evolved during the last several decades into a multidisciplinary model of care. During this time, surgical innovations continued to revolutionize treatment and improve patient outcomes, most notably the introduction of total mesorectal excision (TME) by Heald et al. ${ }^{[1]}$. This landmark discovery changed the trajectory of rectal cancer resections and greatly improved patient outcomes by reducing pelvic recurrences. Since that time, laparoscopic TME was introduced and has

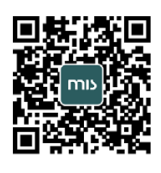


now become standard of care after several large randomized controlled trials (RCTs) assessed oncologic outcomes and early postoperative recovery ${ }^{[2-8]}$.

Performing a laparoscopic TME is not without its challenges, especially in a deep narrow pelvis with the two-dimensional view and limited dexterity. Robotic TME was introduced to overcome some of these challenges. The theoretical technical advantages of robotic TME include a stable camera platform, threedimensional view, and better articulation of the surgical instruments ${ }^{[9]}$. Although this technology has gained widespread popularity, it is not without its own set of challenges, including higher cost, longer operative time, and loss of tactile sensation.

Surgical innovation continues to play a vital role in the multimodal treatment of rectal cancer. Examining the pathologic outcomes is important to ensure appropriate care is provided when introducing new technologies. To date, the largest RCT available to compare laparoscopic and robotic rectal resections is the ROLARR trial ${ }^{[10]}$. Several other RCTs are now available, along with numerous meta-analyses to further evaluate the literature on pathologic outcomes with robotic compared to laparoscopic rectal cancer resections, which are discussed in this review. This review is Part 2 of a two-part series, in which the nononcologic outcomes and learning curve are separately discussed.

\section{Pathologic outcomes}

Total mesorectal excision grade

When assessing pathologic outcomes for rectal cancer, the completeness of the TME is one of the important oncologic factors to consider. It is also a useful marker to compare the effectiveness and safety of the various surgical techniques, such as laparoscopic and robotic. The three RCTs discussed below on robotic vs. laparoscopic approach for the treatment of rectal cancer have assessed TME grade or completeness and none have shown a significant difference in the quality of TME specimen ${ }^{[10-12]}$. Furthermore, multiple metaanalyses have also shown no significant differences.

The ROLAAR RCT trial included TME pathology specimen grading using the method of Quirke and Dixon for completeness and found complete TME in $77.6 \%$ of laparoscopic specimens $v s .76 .4 \%$ of robotic specimens $(P=0.14)^{[10]}$. A phase II open label prospective RCT also assessed the quality of TME by a pathologist, as the primary outcome and found similar results: complete TME 78.1\% (laparoscopic) vs. $80.3 \%$ (robotic) and near complete in $21.9 \%$ (laparoscopic) vs. $18.2 \%$ (robotic) $(P=0.599)^{[11]}$. They did, however, note one incomplete TME $(1.5 \%)$ in the robotic group and none in the laparoscopic group. Lastly, a smaller pilot RCT also found no difference in macroscopic judgement of the TME specimen with complete TME noted in 17 of 18 robotic samples and 1 nearly complete $v s .13$ of 16 complete TME and 3 nearly complete $(P=0.323)^{[12]}$.

A recent meta-analysis by Eltair et al. ${ }^{[13]}$ assessed the robotic and. laparoscopic approaches for the treatment of rectal cancer within nine RCTs that included 1463 patients (728 robotic vs. 735 laparoscopic) ${ }^{[13]}$. Four RCTs were included in the macroscopic assessment of complete TME, including the three discussed above. They found no statistically significant difference in complete resection with zero heterogeneity in their assessment. Simillis et al. ${ }^{[14]}$ compared open $v s$. laparoscopic $v s$. robotic $v s$. transanal mesorectal excision for rectal cancer in their meta-analysis and included 29 RCTs. The authors reported significantly higher incomplete or nearly complete TME in the laparoscopic $v s$. open group (odds ratio of 1.52), but no differences in the laparoscopic vs. robotic (odds ratio of 0.98) approaches. A recent network meta-analysis assessed the quality of TME and reported no difference in complete mesorectum excision in the pooled analysis of 11 studies $^{[15]}$. Nine studies were included in the pooled analysis examining near-complete mesorectal excision and also reported no difference when comparing laparoscopic $v s$. robotic methods. 
One meta-analysis that included 12 studies (11 case-control and only 1 RCT) did find a higher complete TME in robotic vs. laparoscopic surgery (odds ratio of $1.83, P=0.03$ ), however there was significant heterogeneity noted in the analysis $\left(I^{2}=47 \%\right)^{[16]}$. Furthermore, this analysis included a majority of casecontrol studies, which are of lower level of evidence, while other meta-analyses have included more RCTs and prospective studies.

\section{Lymph node harvest}

The current guidelines, including those of the American Joint Committee on Cancer (AJCC) and College of American Pathologists (CAP), recommend a minimum of 12 lymph nodes be examined to accurately stage rectal cancer in order to aid in the decision for adjuvant treatment ${ }^{[17-19]}$. The reasons for low lymph node harvest can include neoadjuvant treatment, lack of high ligation of the vessels, and potentially poor surgical or pathologic technique. When comparing surgical approaches for rectal cancer, it is important to evaluate lymph node harvest with each technique.

The ROLARR RCT performed an intention to treat analysis in which one of the outcomes measured was median lymph nodes retrieved ${ }^{[10]}$. They reported no differences; both groups yielded a high number of lymph nodes: 24.1 (laparoscopic) vs. 23.2 (robotic), almost double the minimal requirement. Kim et al. ${ }^{[11]}$ noted a higher number of lymph nodes in the robotic (median 18) compared to the laparoscopic group (median 15) $(P=0.04)$ in their RCT. They also examined the rate of 12 or more lymph nodes retrieved in their groups and found $90.9 \%$ of patients achieved this benchmark in the robotic group compared to $74 \%$ of patients in the laparoscopic group. Of note, the majority of patients in this single-center RCT received preoperative chemoradiation $(77.3 \%$ in robotic $v s .77 .5 \%$ in laparoscopic), which might have led to the lower number of lymph nodes.

A seven-institution multicenter study examined consecutive patients who underwent robotic or laparoscopic intersphincteric resection for low rectal cancer ${ }^{[20]}$. Propensity score analysis was performed with 1:1 case-match, in which no difference was found in the number of lymph nodes retrieved $(P=0.126)$ or the number of positive lymph nodes $(P=0.712)$. Kim et al ${ }^{[21]}$ also used propensity score matching to analyze their retrospective cohort and, after matching, found no difference in the number of harvested lymph nodes $(P=0.44)$. Furthermore, a propensity score match study was performed in consecutive obese patients who underwent laparoscopic or robotic rectal resection at three centers, and no difference was noted in the mean lymph node yield (17 in robotic $v s .16$ in laparoscopic, $P=0.639)^{[22]}$. A single-center study examined their prospectively collected database of mid to - distal rectal cancers and found a higher median number of lymph nodes harvested (12 in laparoscopic vs. 14 in robotic, $P=0.002)^{[23]}$. However, the groups however were not matched between the median tumor distance of $8 \mathrm{~cm}$ in laparoscopic $v s .7 \mathrm{~cm}$ in robotic. Moreover, there were more male patients, more comorbidities, and preoperative radiation in the robotic surgery group.

Multiple meta-analyses examining the highest level of evidence available in the form of RCTs have found no difference in the number of lymph nodes retrieved when comparing laparoscopic and robotic surgery for rectal cancer ${ }^{[13-15,24,25]}$.

\section{Margins}

Rectal cancer specimen margins assessed are circumferential radial (CRM), proximal, and distal. Ensuring negative margins is of utmost importance in reducing local recurrence rates. Margin assessment is used as a marker to examine and compare surgical techniques. The literature on robotic vs. laparoscopic rectal resection for each margin status is discussed below.

Circumferential Radial Margin: The largest RCT to date on robotic vs. laparoscopic resection for rectal cancer is the ROLARR trial ${ }^{[10]}$. In total, 237 patients were randomized to robotic, of whom the CRM 
status was available for 235 , and 234 to laparoscopic with CRM status available for 224 patients. The CRM positivity rate was $6.3 \%$ in laparoscopic vs. $5.1 \%$ in the robotic group $(P=0.56)$. Kim et al ${ }^{[11]}$ also found similar CRM positivity rates in their RCT with no difference in robotic (6.1\%) compared to laparoscopic (5.5\%) $(P=0.999)$. Eltair et al ${ }^{[13]}$ also confirmed no difference in positive CRM in their pooled analysis of three RCTs in their meta-analysis, but high heterogeneity was noted $\left(I^{2}=57 \%\right)$. Several meta-analyses that included retrospective studies along with the available RCTs have also shown no difference in positive $\mathrm{CRM}^{[14,24-26]}$.

Proximal Resection Margin: Adequate mobilization of the colon, including splenic flexure mobilization, should allow for sufficient proximal resection margins in rectal cancer surgery. The advantages of laparoscopic and robotic rectal cancer resection with this regard pertain to the smaller incisions required for sufficient mobilization compared to open surgery. The three RCTs examined in this review by Jayne et al. ${ }^{[10]}$, Kim et al. ${ }^{[11]}$, and Baik et al. ${ }^{[12]}$ reported no difference in proximal resection margins when comparing robotic to laparoscopic rectal cancer operations. None of the meta-analyses examined in this review reported a difference in proximal margins ${ }^{[13,14,27,28]}$.

Distal Resection Margin: The ROLARR RCT did not compare length of distal margin between the two surgical groups but did note one patient had a positive distal margin in the laparoscopic group ${ }^{[10]}$. Kim et al ${ }^{[11]}$ reported median distal resection margins and noted no statistical difference between robotic $(1.5 \mathrm{~cm})$ and laparoscopic $(0.7 \mathrm{~cm})(P=0.11)$. Baik et al. ${ }^{[12]}$ also noted no difference in mean or median distal resection margins in their groups $(P=0.467)$. Eltair et al. ${ }^{[13]}$ examined five RCTs, which included 455 patients, in their meta-analysis for distal resection margins and found slightly longer distal margins in the robotic group compared to the laparoscopic one with a mean difference of $0.8 \mathrm{~cm}(P=0.004)$. There was significantly high heterogeneity $\left(I^{2}=75 \%\right)$ observed in this pooled analysis. A meta-analysis by Liao et al. ${ }^{[27]}$ included five RCTs, with 340 patients, and also found longer distal margin in the robotic group compared to the laparoscopic one $(P=0.003)$, but again high heterogeneity was noted $\left(I^{2}=75 \%\right)$. Simillis et al. ${ }^{[14]}$ also found the robotic surgical approach to have higher distal resection margins when compared to open (7.6 $\mathrm{mm})$, laparoscopic $(6.8 \mathrm{~mm})$, and transanal $(6.8 \mathrm{~mm})$ techniques. There were no reported data on positive distal margins for any of these groups.

\section{CONCLUSION}

Introduction of new surgical techniques to further surgical innovation and improve patient outcomes should be judiciously undertaken to ensure patient care, most notably that oncologic outcomes are not compromised. The majority of the high-level available evidence has found no differences between the two surgical approaches relative to TME completeness, lymph node harvest, positive CRM, or proximal resection margin. A longer distal resection margin has been found with robotic compared to laparoscopic approaches in meta-analyses, but not in RCTs. However, there is no evidence that a longer distal margin translates to better oncological outcomes.

Based on the current literature, either approach, laparoscopic or robotic, is safe and effective from a pathology standpoint. Since the two techniques are comparable, other outcomes and factors need to be considered when recommending one versus the other to our patients. The non-pathology outcomes are discussed in a separate review and should be strongly considered ${ }^{[29]}$.

Scrutinizing ones' own rectal cancer resection outcomes is even more important than reviewing the literature. The Commission on Cancer's National Accreditation Program for Rectal Cancer was established to ensure the highest quality metrics based on the highest level of evidence available are followed ${ }^{[30]}$. The NAPRC requires data collection and monitoring, which should help the provision of optimal care. A national program of this caliber allows for further tracking of current care processes to better evaluate 
the care model to continue improving patient care. Regardless of what surgical technique is chosen by the surgeon, a multidisciplinary team approach must be applied to optimize oncologic outcomes ${ }^{[31,32]}$.

\section{DECLARATIONS}

Authors' contributions

Manuscript preparation: Ghuman A, Kavalukas S

Manuscript review and editing: Wexner SD

\section{Availability of data and materials}

Not applicable.

\section{Financial support and sponsorship}

None.

\section{Conflicts of interest}

Dr. Wexner is a paid consultant and receives royalties for intellectual property license from Intuitive Surgical, Medtronic, and Karl Storz and is a paid consultant for Stryker, Takeda, and Baxter. All other authors declared that there are no conflicts of interest.

\section{Ethical approval and consent to participate}

Not applicable.

\section{Consent for publication}

Not applicable.

\section{Copyright}

(c) The Author(s) 2020.

\section{REFERENCES}

1. Heald RJ, Husband EM, Ryall RD. The mesorectum in rectal cancer surgery--the clue to pelvic recurrence? Br J Surg 1982;69:613-6.

2. Fleshman J, Branda M, Sargent DJ, et al. Effect of laparoscopic-assisted resection vs open resection of stage II or III rectal cancer on pathologic outcomes. JAMA 2015;314:1346-55.

3. Fleshman J, Branda ME, Sargent DJ, et al. Disease-free survival and local recurrence for laparoscopic resection compared with open resection of stage II to III rectal cancer: follow-up results of the ACOSOG Z6051 randomized controlled trial. Ann Surg 2019;269:589-95.

4. Bonjer HJ, Deijen CL, Abis GA, et al. COLOR II Study Group. A randomized trial of laparoscopic versus open surgery for rectal cancer. N Engl J Med 2015;372:1324-32.

5. van der Pas MH, Haglind E, Cuesta MA, et al. Laparoscopic versus open surgery for rectal cancer (COLOR II): short-term outcomes of a randomised, phase 3 trial. Lancet Oncol 2013;14:210-8.

6. Stevenson AR, Solomon MJ, Lumley JW, et al. ALaCaRT Investigators. Effect of laparoscopic-assisted resection vs open resection on pathological outcomes in rectal cancer: the ALaCaRT randomized clinical trial. JAMA 2015;314:1356-63.

7. Kang S, Park JW, Jeong S, et al. Open versus laparoscopic surgery for mid or low rectal cancer after neoadjuvant chemoradiotherapy (COREAN trial): short-term outcomes of an open-label randomised controlled trial. Lancet Oncol 2010;11:637-45.

8. Jeong S, Park JW, Nam BH, et al. Open versus laparoscopic surgery for mid-rectal or low-rectal cancer after neoadjuvant chemoradiotherapy (COREAN trial): survival outcomes of an open-label, non-inferiority, randomised controlled trial. Lancet Oncol 2014;15:767-74.

9. Holder-Murray J, Dozois EJ. Minimally invasive surgery for colorectal cancer: past, present, and future. Int J Surg Oncol 2011;2011:490917.

10. Jayne D, Pigazzi A, Marshall H, et al. Effect of robotic-assisted vs conventional laparoscopic surgery on risk of conversion to open laparotomy among patients undergoing resection for rectal cancer: the ROLARR randomized clinical trial. JAMA 2017;318:1569-80.

11. Kim MJ, Park SC, Park JW, et al. Robot-assisted versus laparoscopic surgery for rectal cancer: a phase II open label prospective randomized controlled trial. Ann Surg 2018;267:243-51.

12. Baik SH, Ko YT, Kang CM, et al. Robotic tumor-specific mesorectal excision of rectal cancer: short-term outcome of a pilot randomized trial. Surg Endosc 2008;22:1601-8. 
13. Eltair M, Hajibandeh S, Hajibandeh S, et al. Meta-analysis and trial sequential analysis of robotic versus laparoscopic total mesorectal excision in management of rectal cancer. Int J Colorectal Dis 2020;35:1423-38.

14. Simillis C, Lal N, Thoukididou SN, et al. Open versus laparoscopic versus robotic versus transanal mesorectal excision for rectal cancer: a systematic review and network meta-analysis. Ann Surg 2019;270:59-68.

15. Rausa E, Bianco F, Kelly ME, et al. Systemic review and network meta-analysis comparing minimal surgical techniques for rectal cancer: quality of total mesorectum excision, pathological, surgical, and oncological outcomes. J Surg Oncol 2019;119:987-98.

16. Milone M, Manigrasso M, Velotti N, et al. Completeness of total mesorectum excision of laparoscopic versus robotic surgery: a review with a meta-analysis. Int J Colorectal Dis 2019;34:983-91.

17. Compton CC, Greene FL. The staging of colorectal cancer: 2004 and beyond. CA Cancer J Clin 2004;54:295-308.

18. Compton CC, Fielding LP, Burgart LJ, et al. Prognostic factors in colorectal cancer. College of American Pathologists Consensus Statement 1999. Arch Pathol Lab Med 2000;124:979-94.

19. Sobin LH, Greene FL. TNM classification: clarification of number of regional lymph nodes for pn0. Cancer 2001;92:452.

20. Park JS, Kim NK, Kim SH, et al. Korean Laparoscopic Colorectal Surgery Study Group. Multicentre study of robotic intersphincteric resection for low rectal cancer. Br J Surg 2015;102:1567-73.

21. Kim J, Baek SJ, Kang DW, et al. Robotic resection is a good prognostic factor in rectal cancer compared with laparoscopic resection: long-term survival analysis using propensity score matching. Dis Colon Rectum 2017;60:266-73.

22. Panteleimonitis S, Pickering O, Abbas H, et al. Robotic rectal cancer surgery in obese patients may lead to better short-term outcomes when compared to laparoscopy: a comparative propensity scored match study. Int J Colorectal Dis 2018;33:1079-86.

23. Law WL, Foo DCC. Comparison of short-term and oncologic outcomes of robotic and laparoscopic resection for mid- and distal rectal cancer. Surg Endosc 2017;31:2798-807.

24. Hoshino N, Sakamoto T, Hida K, Sakai Y. Robotic versus laparoscopic surgery for rectal cancer: an overview of systematic reviews with quality assessment of current evidence. Surg Today 2019;49:556-70.

25. Gavriilidis P, Wheeler J, Spinelli A, de'Angelis N, Simopoulos C, Di Saverio S. Robotic vs laparoscopic total mesorectal excision for rectal cancers: has a paradigm change occurred? A systematic review by updated meta-analysis. Colorectal Dis 2020; doi: 10.1111/ codi. 15084 .

26. Zheng B, Zhang X, Wang X, et al. A comparison of open, laparoscopic and robotic total mesorectal excision: trial sequential analysis and network meta-analysis. Colorectal Dis 2020;22:382-91.

27. Liao G, Zhao Z, Deng H, Li X. Comparison of pathological outcomes between robotic rectal cancer surgery and laparoscopic rectal cancer surgery: a meta-analysis based on seven randomized controlled trials. Int J Med Robot 2019;15:e2027.

28. Lee SH, Kim DH, Lim SW. Robotic versus laparoscopic intersphincteric resection for low rectal cancer: a systematic review and metaanalysis. Int J Colorectal Dis 2018;33:1741-53.

29. Kavalukas SL, Ghuman A, Sharp SP, Wexner SD. Robotic or laparoscopic surgery for rectal cancer - which is the best answer? a comprehensive review of non-oncological outcomes and learning curve. Mini-invasive Surg 2020;4:61.

30. American College of Surgeons. National Accreditation Program for Rectal Cancer. Available from: https://www.facs.org/qualityprograms/cancer/napre\#. [Last accessed on 9 Nov 2020]

31. Richardson B, Preskitt J, Lichliter W, et al. The effect of multidisciplinary teams for rectal cancer on delivery of care and patient outcome: has the use of multidisciplinary teams for rectal cancer affected the utilization of available resources, proportion of patients meeting the standard of care, and does this translate into changes in patient outcome? Am J Surg 2016;211:46-52.

32. Karagkounis G, Stocchi L, Lavery IC, et al. Multidisciplinary conference and clinical management of rectal cancer. J Am Coll Surg 2018;226:874-80. 